\title{
Experiencia Docente en Interconsulta psiquiátrica en Atención Primaria de Salud para residentes.
}

\author{
Teaching experience in Psychiatric Consultant-laison \\ in Primary Care Settings.
}

Julia Acuña, Arturo Roizblatt, Claudio Fullerton y Alberto Botto.

Departamento de Psiquiatría y Salud Mental. Sede Oriente, Facultad de Medicina. Universidad de Chile.

Se describe el proceso de implementación de programa de Interconsulta psiquiátrica docente para residentes, realizado por el Departamento de Psiquiatría y Salud Mental de la Facultad de Medicina de la Universidad de Chile entre 2003 y 2004.

Método: Se establece programa preliminar, con objetivos y pautas de evaluación de cumplimiento de objetivos por parte de residentes.

Resultados: Se destaca evaluaciones en el rango superior que los residentes hacen de objetivos docentes. Más bajos fueron evaluados los objetivos asistenciales. Supervisores evalúan positivamente el proceso en general, identificando las principales dificultades observadas: entre ellas la doble agenda presente en el proceso complejo propio de la actividad y de los objetivos docentes.

Se destaca la importancia de la supervisión docente, la cual debería ser activa, vigilar las áreas que obstaculizan el proceso; mayores dificultades provenientes de demandas implícitas o explícitas subyacentes operaron como resistencias en los profesionales de salud general:"demandas urgentes"

\footnotetext{
Correspondencia:

J. Acuña,

Av. Salvador $N^{\circ} 486$

Providencia

Santiago de Chile. S.A.

Fono-fax (56)(2) 2748855

e-mail: jacuna@med.uchile.cl
}

y "pacientes emocionalmente sobrepasados" postergarían la adecuada y oportuna acogida y tratamiento de los problemas de salud mental presentados. Tanto el docente - supervisor como el residente deberían advertir oportunamente estas resistencias para ayudar a superar estas barreras; entonces el profesional de salud general sería capaz de acoger y responder a las demandas emocionales explícitas o implícitas presentadas en la entrevista.

Conclusión: No obstante la complejidad del proceso descrito, la interconsulta psiquiátrica es muy bien valorada por todos los actores involucrados en proveer atención a pacientes que presentan problemas de salud mental en servicios de salud general.

Palabras claves: Interconsulta psiquiátrica, residentes, atención primaria, salud mental.

Authors describe an initial program for training residents as consultants in Primary Care Settings. This program was developed and applied by the Department of Psychiatry and Mental Health of the Faculty of Medicine of the Universidad de Chile between 2003 and 2004.

Method: Preliminary program was established, with objectives and evaluation schemes for the correct achievement of the objectives by the residents in two areas: learning and clinical work. Residents were assigned to different primary health centers, under a supervisor.

Results: Good evaluations (superior rank) for residents' achievements in learning objectives were observed. 
Lower ratings were for clinical objectives.

Supervisors evaluated the whole process positively; the main difficulty mentioned was the double work schedule required in the whole process of Psychiatry Consultant-laison in Primary settings.

The discussion emphasizes the importance of supervision, which should be active in watching areas that hinder the process; making it necessary to detect problems in need of change to be established as soon as possible. Greater difficulties were originated on explicit and/or underlying medical or general health care team resistances. "Urgent demands" and emotionally overwhelming patients delayed competent reception and treatment. Supervisor and resident should offer proper and timely advice, to allow the overcoming of the difficulties, then general health professionals would be able to manage the emotional demands, explicit or tacit, presented in the interview. Some elements should be treated in the process, itself complex, of teaching consultant-liaison during the psychiatry resident program.

Conclusion: Nevertheless, psychiatry consultant-liaison in primary care settings was highly appreciated as necessary by all the actors involved in providing care for patients presenting mental health problems in primary health care settings

Key words: psychiatry consultant-liaison, residents, primary care, mental health.

\section{INTRODUCCIÓN}

Existe una amplia literatura en el tema de Interconsulta psiquiátrica en relación a servicios clínico-hospitalarios, destacando la importancia del tema derivada de las altas prevalencias de trastornos mentales, la alta comorbilidad y el impacto negativo en la evolución clínica y en costos asocia$\operatorname{dos}^{1-5}$. Estos hallazgos son similares a los revelados en centros de salud general ${ }^{6-9}$; sin embargo, las publicaciones generales acerca de la Interconsulta psiquiátrica en Atención Primaria de Salud (APS) son escasas y más aún, aquellas que hacen referencia al proceso de enseñanza requerido, el cual sólo es aludido de modo tangencial.

El trabajo de enlace del especialista en psiquiatría con el equipo de salud general forma parte del mode- lo de atención sanitaria propuesta por la Organización Mundial de la Salud (OMS). Junto con reconocer que " 1 de cada 4 personas sufre un problema de salud mental", establece entre las 10 recomendaciones para salud mental "...dispensar tratamiento en Atención Primaria de acuerdo al escenario local..."10. En Chile, donde hay cifras de prevalencia de consulta en Atención Primaria del 50\%, el Plan Nacional de Salud Mental ha incluido recientemente la interconsulta psiquiátrica como metodología para el buen desarrollo de esta estrategia ${ }^{11}$. Se requiere por tanto, que los especialistas trabajen fluida y eficientemente en la interfase con los equipos de salud primario. Resulta entonces indispensable que la formación del psiquiatra, considere el desarrollo de habilidades y destrezas que permitan tales desempeños. No obstante, el conocimiento en el tema es limitado y la gran variabilidad de las publicaciones no permite disponer de principios definitivos sobre los cuales apoyar los programas de residencia en estas materias. ${ }^{12-16}$

Este trabajo tiene como objetivo describir y evaluar el proceso de implementación de docencia en interconsulta psiquiátrica para residentes de psiquiatría, desarrollado por el Departamento de Psiquiatría y Salud Mental Oriente (DPSMO) de la Facultad de Medicina de la Universidad de Chile en consultorios de APS del área Oriente de Santiago de Chile.

\section{MATERIAL Y MÉTODO}

El DPSMO de la Facultad de Medicina de la Universidad de Chile en coordinación con la Unidad de Salud Mental del Servicio de Salud Metropolitano Oriente de Santiago de Chile (SSMO) asumió en el año 2002 la interconsulta psiquiátrica de cinco Centros de Salud Familiar (CESFAM) distribuidos en 3 comunas del sector Oriente de Santiago.

La interconsulta psiquiátrica se ha definido como "un encuentro de trabajo programado en el cual se reúnen profesionales de Salud Primaria con un psiquiatra o especialista de Salud Mental, destinada a mejorar la capacidad resolutiva y el manejo adecuado de los trastornos psiquiátricos atendidos en APS" tal como es planteado por el Ministerio de Salud (MINSAL) de Chile ${ }^{11}$. Se trata por tanto de una actividad esencialmente de capacitación, orientada a desarrollar habilidades y destrezas clínicas para que el médico general y el equipo de salud general, brinden los servicios de salud mental apropiados a la complejidad de su competencia.

El programa de formación de especialistas en Psiquiatría de la Facultad de Medicina de la 
TABLA 1. Objetivos del programa de consultoría docente de psiquiatria y salud mental. Departamento de Psiquiatría y Salud Mental Facultad de Medicina - Campus Oriente. Universidad de Chile

\section{OBJETIVOS DOCENTES}

\begin{tabular}{ll}
\hline GENERAL & Incorporar al médico en formación en la especialidad al trabajo en red en salud mental a través \\
del ejercicio de interconsulta psiquiátrica, según objetivos formativos y asistenciales propuestos
\end{tabular}

ESPECÍFICOS 1) Desarrollar habilidades y destrezas para trabajar en equipo

2) Desarrollar capacidad de liderazgo del psiquiatra en formación en los diferentes dispositivos de la red de salud mental local.

3) Identificar las ventajas y dificultades del trabajo en red de salud mental

4) Aplicar conocimientos teóricos en la elaboración y ejecución de un programa local de salud mental

5) Desarrollar soluciones a diferentes problemas detectados en la consultoría

6) Realizar diagnóstico de situación, identificando necesidades específicas.

7) Formular un programa de consultoría

8) Elaborar informe final de consultoría

\section{OBJETIVOS ASISTENCIALES}

GENERAL $\quad$ Fortalecer la calidad la calidad de los servicios otorgados en la red de salud mental del Servicio de Salud metropolitano oriente, a través de una atención oportuna y de acuerdo a las mejores prácticas clínicas.

ESPECÍFICOS 1) Contribuir a aumentar el nivel de resolutividad de equipos de APS en manejo de patología psiquiátrica más prevalente y prioritarios a nivel local: depresión y trastornos psiquiátricos severos.

2) Facilitar y mejorar el sistema de referencia y contra- referencia de pacientes con patología psiquiátrica y/o problemas de salud mental atendidos en consultorios asignados.

Universidad de Chile, se imparte a lo largo de 3 años. El plan de formación establece asignaturas teóricas, y asignaturas teórico prácticas. Entre estas últimas, está la signatura de Psiquiatría Social y Salud Mental. Consta ésta de un curso teórico-práctico, impartido en el primer año de la formación, y una práctica anual de interconsulta psiquiátrica, en segundo o tercer año, con horarios parciales adecuados a la necesidades y disponibilidad del equipo de salud primaria de la Red del Servicio de Salud Metropolitano Oriente.

Se distribuyeron los residentes en los CESFAM, asignándose un psiquiatra docente a cada uno de ellos. El trabajo de esta primera experiencia de con- sultoría fue realizado por residentes de psiquiatría de segundo y tercer año entre Mayo de 2002 y Marzo de 2004. Consideró actividades en los consultorios de frecuencia variable ( 1 a 2 veces por mes) de 90 o 120 minutos de duración cada una. Se programaron dos modalidades de supervisión: una directa durante la actividad en el consultorio y otra entre residente y supervisor fuera del consultorio.

Para desarrollar la actividad se estableció un programa preliminar con objetivos, requisitos mínimos para desarrollar la consultoría, evaluación y definiciones operacionales (Tablas 1 y 2 ). Entre las posibles actividades a desarrollar se incluyó: seguimiento de casos, reuniones clínicas y capacitación teórica.

TABLA 2. Definiciones operacionales del programa docente de interconsulta psiquiàtrica en APS.

Departamento de Psiquiatría y Salud Mental Facultad de Medicina - Campus Oriente. Universidad de Chile

CONSULTORÍA PSIQUIATRICA Encuentro de trabajo que reúne en el Consultorio o Centro de Salud al equipo de Salud General (APS) con el especialista (del nivel secundario). El encuentro debe ser programado con el tiempo adecuado a la actividad; debe ser regular y tener la frecuencia de acuerdo a objetivos convenidos entre las partes.

CONSULTORIA TÉCNICA

CAPACITACIÓN
Revisión de criterios diagnósticos y terapéuticos de acuerdo a complejidad de casos y guías clínicas vigentes. Consulta aspectos teóricos y prácticos. Tratamiento integral de cada patología. ( Por Ej.: depresión señalado en Programa MINSAL Chile).

Sesiones teorico-prácticas destinadas a desarrollar conocimientos teóricas, habilidades y destrezas en torno a principales temas en uso o por desarrollar de acuerdo a necesidades locales y a normas técnicas vigentes. 
TABLA 3. Evaluación de Objetivos de Consultoría realizada por residentes del Programa de formación en Psiquiatría. 2002-2004. ( $n=10)$. Departamento Psiquiatría. Sede Oriente. Facultad de Medicina. Universidad de Chile.

OBJETIVOS DOCENTES

Incorporar al residente al trabajo en red

Desarrollar habilidades y destrezas para trabajo en equipo .

Desarrollar capacidad de liderazgo..

Identificar ventajas y dificultades del trabajo en red

Aplicar conocimientos teóricos acerca de elaboración y ejecución de programas de salud mental...

Desarrollar soluciones a diferentes problemas detectados en consultoría

Realizar diagnóstico de situación .

Formular programa de consultoría

Elaborar informe final
Nota promedio

(mínima 1-máxima 7)

$6,1(4-7)$

$.6,2(5-7)$

$.5,7(3-7)$

$6,2(5-7)$

$. .5,8(4-7)$

$.5,9(4-7)$

$6,0(4-7)$

$.6,4(5-7)$

$6,4(5-7)$

OBJETIVOS ASISTENCIALES

Fortalecer calidad de servicios entregados en la Red de Salud Mental

$5,5(2-7)$

Contribuir a mejorar el nivel de resolutividad de equipos de APS en problemas de Salud Mental más prevalentes...................5,9 (2-7)

Facilitar y mejorar sistema de referencia y contra-referencia de pacientes psiquiátricos atendidos. ..............................................5,6 (4-7)

Para la evaluación, se diseñó una encuesta autoadministrada para residentes, quienes debían completarla al final del periodo. La encuesta constaba de dos partes, una cuantitativa y otra cualitativa; allí, los residentes evaluaron el grado de cumplimiento de los objetivos (docentes y asistenciales) en escala de 1 a 7 , y los aspectos más y menos valiosos de la experiencia docente a través de preguntas abiertas. (Tabla 3 ).

Los docentes realizaron supervisiones una vez al mes con el (los) residente(s), para analizar las dificultades observadas, identificar necesidades docentes y se planificaron las sesiones de consultoría. Posterior a ello los supervisores, se reunieron cada tres meses para revisar y poner en común el proceso de implementación de la consultoría. Estas dos actividades se realizaron sin pautas estructuradas previamente. Se obtuvo así una evaluación de carácter cualitativo, que en esta etapa tenía como principal objetivo identificar las necesidades docentes y las dificultades del proceso en marcha.

\section{RESULTADOS}

\section{Evaluación realizada por residentes.}

Diversos aspectos resultaron de interés en la evaluación realizada por los residentes. En general, los residentes consideraron que los objetivos docentes fueron mejor cumplidos que los asistenciales. Entre ellos destacan: desarrollar habilidades y destrezas para trabajo en equipo, identificar ventajas y dificultades del trabajo en red e incorporar al residente al trabajo en red. Entre los objetivos asistenciales, el fortalecimiento de la calidad de los servicios entregados, como la facilitación y mejoramiento de los sis- temas de referencia y contra-referencia de pacientes psiquiátricos, resultaron con las evaluaciones más deficientes (Tabla 3 ).

La evaluación cualitativa destaca entre los aspectos positivos "poder conocer el funcionamiento en la red" Entre los elementos negativos, se menciona "falta de tiempo del equipo APS", la cual es observada como un obstáculo al proceso formativo por parte de los residentes. La supervisión docente es evaluada como "buena" y "planificada"; sin embargo se percibe como insuficiente, especialmente al inicio.

\section{Evaluación realizada por docentes -supervisores}

2.1. Apreciación general del proceso.- Se observó cierto grado de inquietud y ansiedad en los residentes al inicio del proceso. Una vez efectuadas las primeras sesiones, los residentes adquirieron autonomía y tomaron independencia, abordando dos líneas de trabajo: supervisión de casos y sesiones de capacitación. A medida que avanzaba el programa de consultoría, se observó a los residentes más seguros e involucrados en la actividad.

En general en esta primera experiencia hubo mayor preocupación por los objetivos asistenciales de la consultoría en desmedro de otros objetivos docentes de la actividad.

\subsection{Dificultades derivadas de la realidad local de CESFAM, su dinámica y características.}

Destaca la participación irregular de los profesionales de APS en las actividades programadas, a pesar del interés manifiesto y buena disposición de parte de los directivos locales para dar facilidades e 
incorporar las consultorías en la programación general. La alta rotación de profesionales observada en APS, especialmente médicos y psicólogos, obliga a repetir actividades o temas.

\section{DISCUSIÓN}

La actividad fue evaluada positivamente por todos los actores involucrados, haciendo referencia a la importancia y alta prevalencia que los temas de salud mental tienen en la consulta de salud general y, a las necesidades de diverso tipo que de ello se desprenden.

Si bien el presente trabajo se refiere a un proceso inicial de 2 años de duración, y con una muestra reducida de 10 residentes, pensamos que constituye un aporte a la escasa literatura que se ha ocupado del tema.

Un aspecto central de este proceso enseñanzaaprendizaje, lo constituye la doble agenda que es necesario desarrollar de modo simultáneo ${ }^{12,15}$; se trata de:

- aquella referida a la capacitación teórica acerca de tópicos específico

- aquella orientada a abordar los aspectos emocionales explícitos e implícitos de la relación asistencial en la consulta de salud general.

Respecto de la primera, se observó que esta función era asumida por el residente con bastante facilidad; además el M.G. sintonizaba fácilmente con ella.

Respecto de la segunda agenda, el M.G. estuvo menos dispuesto, mostrando ciertas reticencias. Pensamos que los residentes no alcanzaron a superar esta barrera, que limita las posibilidades de enseñar habilidades y destrezas para el abordaje de los problemas de salud mental presentes en la consulta. Sólo así será posible que este proceso de aprendizaje más complejo y menos obvio, pueda darse en los profesionales. La consultoría debe enfocar y superar estas dificultades de modo proactivo, enfatizando objetivos al respecto.

A continuación se ordena la discusión en torno a cuatro puntos que en nuestra experiencia resultaron de mayor relevancia:

\section{CAPACITACIÓN PRELIMINAR}

\section{DEL RESIDENTE}

En el proceso enseñanza-aprendizaje evaluado se destacó la importancia que tiene para los residentes disponer de una capacitación específica previo al inicio de la actividad. Como ha sido destacado por algunos autores, el rol del consultor debe estar per- fectamente definido ${ }^{12-17}$ En el caso de los residentes esto cobra mayor relevancia pues a través de ella pueden distinguir los objetivos y posteriormente orientarse hacia ellos. Además se debería permitir que el residente se familiarice con las características de los centros de salud donde se desempeñará como consultor; para ello en la capacitación se debería incluir aspectos tales como:

- anticipar posibles escenarios y situaciones a las cuales se verá enfrentado

- saber de la alta rotación de profesionales

- saber sobre la discordancia entre el discurso y la práctica respecto de la importancia que efectivamente se da a la consultoría por parte del equipo APS, lo cual se manifiesta a través de impuntualidad o inasistencia de los profesionales a las actividades.

Si bien estos aspectos pudieran ser propios de la realidad chilena, nos parece que algunas de estas conductas pueden ser manifestación de resistencias más o menos inconscientes de parte del equipo de salud y por tanto, comunes a otras realidades.

Un elemento central de la capacitación debería considerar talleres orientados al desarrollo de destrezas adecuadas a los objetivos propuestos. En la formación de Psiquiatría tradicional, no se considera suficientemente la enseñanza de habilidades para el manejo de situaciones emocionales difíciles, que le permitan al M.G., tener acceso a contenidos implícitos presentes en la consulta de salud general. Temas como manejo de grupos, enseñanza de intervenciones psicoterapéuticas inespecíficas, deberían ser talleres ofrecidos durante la capacitación de los residentes ${ }^{12,15,17,18,19}$.

\section{EL PROCESO}

Resultó fundamental el inicio formal de la actividad con la participación de los jefes administrativos y los participantes de la actividad. La interconsulta psiquiátrica se insertó así claramente en las actividades regulares de los profesionales de APS. Creemos que esto puede dar cuenta, al menos parcialmente, de los buenos resultados observados, como ha sido señalado en la literatura ${ }^{12,13,20}$.

Corroboramos lo planteado por algunos autores respecto del rol primordial que juega el consultor, en este caso residente - consultor, para el éxito de la actividad, quien debe ser capaz de captar y valorar cabal y genuinamente la realidad y características del trabajo realizado en APS y de los profesionales involucrados ${ }^{12,15,17,20,21,22,23,24}$. Se podrá desarrollar así un trabajo de equipo que eficientemente responda a las necesidades de salud mental de los pacientes. 


\section{LA SUPERVISIÓN}

Nos pareció que el rol del supervisor fue fundamental. En nuestro trabajo se puso de manifiesto que la mayor dificultad en este proceso docente - asistencial consiste en superar las dificultades locales de la consultoría y las resistencias presentadas. Superadas estas barreras, será posible abordar efectivamente los elementos emocionales de la consulta de salud mental, para lo cual se debe motivar y desarrollar habilidades en los profesionales de APS. Pensamos que estos tres elementos, manifestados a través de distintos procesos, podrán ser mejor resguardados disponiendo de una pauta de supervisión estructurada en base a los objetivos correspondientes. Especial importancia se debe asignar a las supervisiones directas orientadas a estos fines ${ }^{9,22,23,24}$.

La práctica de la supervisión nos mostró que el docente supervisor tiene que adecuar, junto con el residente, los objetivos docentes específicos de acuerdo a las características del residente y de la realidad en la cual se desarrolla la consultoría.

\section{LA EVALUACIÓN}

La evaluación de todo proceso enseñanza-aprendizaje constituye el pilar del mejoramiento y adecuación permanente del mismo. Algunos autores la han destacado en relación a consultoría, aludiendo a cualidades especificas ${ }^{12,15,25}$. La evaluación de esta primera experiencia docente se orientó hacia los objetivos asistenciales y docentes, según la visión de los residentes. Esta es una limitación que deberá ser mejorada en el programa. En relación a objetivos asistenciales y docentes en esta primera experiencia se incluyó sólo una evaluación global de la valoración del trabajo de consultoría en los diferentes temas abordados. Se requiere de elementos más específicos, en términos de los logros esperados en los profesionales de APS y en los residentes. También pensamos que la evaluación de los residentes a sus docentes supervisores fue muy general y debería ser perfeccionada.

\section{RECOMENDACIONES FINALES}

Nos parece de interés destacar como resultado de esta experiencia, que el trabajo de consultoría docente debería considerar a lo menos los siguientes elementos:

- Los residentes de psiquiatría deberían tener un período de capacitación específica, antes de iniciar la consultoría. En ella, y a modo de proceso de inducción, conocerían las herramientas básicas para enfrentar las dificultades propias de una dinámica compleja como la descrita y la realidad en la cual se desempeñarán.

- El proceso enseñanza/aprendizaje acá señalado, es de alta complejidad y como tal requiere una particular dedicación docente. La supervisión constituye la actividad docente esencial, a través de la cual se debe resguardar permanentemente que:

- Se alcancen mejores desempeños del equipo de APS en el diagnóstico y tratamiento de los pacientes psiquiátricos, $\mathrm{y}$

- el residente desarrolle habilidades para trabajar en equipo, ejercer liderazgo en el grupo y tenga claridad respecto de cual desempeño se espera de él: - no se trata solamente que "trasmita conocimientos", sino también debe ser capaz de aclarar y entusiasmar a los profesionales de APS respecto de la importancia del trabajo por ellos realizado

- debe trasmitir al equipo de salud general que tanto o más importante que los conocimientos son los elementos emocionales explícitos o tácitos en la entrevista con un paciente con trastorno psiquiátrico o problemas de salud mental.

- su énfasis permanente será permitir el desarrollo de habilidades y destrezas lo cual debería ser señalado de modo claro y constituir un tema relevante de la consultoría.

- Se debe disponer y adecuar de modo permanente los métodos de evaluación de los diferentes aspectos incluidos en este complejo proceso de enseñanzaaprendizaje.

Finalmente destacamos la necesidad de mayores experiencias, aunque la complejidad del proceso referido requerirá de la adecuación de los métodos a la realidad local.

\section{BIBLIOGRAFÍA}

1. Ruddy $\mathrm{R}$ y House A. Meta-review of high-quality systematic reviews of interventions in key areas of liaison psychiatry. $\mathrm{Br}$ J of Psychia. 2005; 187:109-120

2. Davies M. Towards the develpment of a reciprocal liaison service. Psychiatrc Bulletin. 2000; 24:379-381.

3. Hall RC y Frankel GL. The value of consultation-liaison interventions to the general hospital. Psychiatric Services. 1996; 47:418-420

4. Smith GR, Rost K y Kashner TM. A trial of the effect of a standarised psychiatric consultation on health outcomes and costs in somatising patients. Archives of General Psychiatry. 1995; 52: $238-243$

5. Strai Jj, Smith GC, Hammer JS, Mackenzie DP, Blummenfield M y cols. Adjustement Disorder: a multisite study of its utili- 
zation and interventions in the Consultation-Liaiso Psychiatry. Gen Hosp Psichiatry. 1998; .....

6. Main D, Lutz L, Barret J, Mathew J, Miller R. The role of primary care clinician attitudes, beliefs and training in the diagnosis and treatment of Depression. Arc Fam Med. 1993; 2: 10611066.

7. Molodynski A, Bolton J, Guest L,. Is liaison psychiatry a separate specialty? Comparison of referrals to a liaison psychiatry servcie and a community mental health. Psychiatric Bulletin. 2005; 29: 342-345.

8. Katon W, Von Korf M, Lin E et al. A randomized trial of psychiatric consultation with distressed high utilizers of health care. Gen Hosp Psychiatry. 1992; 14: 86-98.

9. Simon GE, Von Korf M, Barlow W. Somatization and psichiatric disorders in the NMIH Epidemiologic Catchment Area Study. Am J Psychiatry. 1991;148: 1494-1500.

10. Informe sobre la salud en el mundo 2001: Salud Mental: nuevos conocimientos, nuevas esperanzas. OMS. Ginebra. Suiza. 2001.

11. Plan Nacional de Salud Mental. MINSAL. Chile. 2000.

12. Cowley D, Katon W, Veith R. Training psychiatry residents as consultants in Primary Care Settings. Academic Psychiatry. 2000; 24(3):124-132 .

13. Silberman E. Should we train psychiatrists as primary care providers?. Psychosomatics 1999; 40: 126-129.

14. Hales RE. Primary care in psychiatry residency training. Gen Hosp Psychiatry 1980; 2(2):148

15. Yudkowsky R. So you want to train Psychiatry residents in ambulatory primary care settings?. Academic psychiatry 2000; 24(3):133-138.

16. Tohyama T, Yamashita N. Guidance of community menthal health training for psychiatry residents. Nippon Koshu Eissei Zasshi. 1993; 40(11):1062-8.

17. Gask L, Croft J. Methods of working with primary care. Advances in psychiatric Treatment. 2000; 6: 442-449.

18. Mcculloch J, Ramesar S, Peterson H. Psychoterapy in Primary Care: The BATHE technique. Disponible en: http:// www.aafp.org/afp/980501/ap/mccullo... accesado 17-12-2004.

19. Montgomery V. Psychotherapy: Primary care providers = partner in treating depression. Disponible en:

http://www.dcmsonline.org/iax-medicine/2002journals.... accesado 22-09-2004.

20. Thompson E, Margo G. Psychiatry and family medicine: Better training is the key for better professional cooperation. Academic Psychiatry. 1995;19:224-226

21. Rozan G, Holmes D. Evaluating the impact of the psychiatric course for non psychiatric physicians. Ment Hyg 1968; 52:612616.

22. Freeland A, Levine S, Johnston M, Busby K. Training residents for community psychiatric practice: the resident perspective. Can J Psichiatry. 2000; 45(7):655

23. Hodges B, Inch C, Silver I. Improving the psychiatric knowledge, skills and attitudes of Primary Care Physicians, 19502000: a review. Am J Psychiatry. 2001; 158: 1579-85.

24. Godgins G, Judd F, Kyrios M. Murray G, et als. Psychiatry and general practice A mode of supervision in mental health for general practitioners. Australasian Psychiatry. 2005; 13(2). Disponible en http://www.blackwell-synergy.com/doi/abs/ accesado 13-06-2005.

25. Gask L, Sibbald B, Creed F. Evaluating models at the interface between mental health services and primary care. The British Journal of Psychiatry. 1997; 170: 6-11. 